\title{
Levels of brain natriuretic peptide as a marker for the diagnosis and prognosis of acute ischemic stroke
}

Saadet Sayan ${ }^{1}$, Dilcan Kotan ${ }^{2}$

${ }^{1}$ Department of Neurology, SB Sakarya University Research and Training Hospital, Sakarya, Turkey

2Department of Neurology, Faculty of Medicine, Sakarya University, Sakarya, Turkey

Submitted: 10 March 2016

Accepted: 15 April 12016

Arch Med Sci Atheroscler Dis 2016; 1: e16-e22

DOI: 10.5114/amsad.2016.59751

Copyright @ 2016 Termedia \& Banach

\section{Abstract}

Introduction: The relationships between plasma levels of brain natriuretic peptide (BNP) and severity and location of stroke, prognosis, and infarct volume were investigated in acute ischemic stroke patients who presented within the first 24 hours (h) of stroke.

Material and methods: Brain natriuretic peptide levels were tested in $40 \mathrm{pa}$ tients and 30 healthy controls. Infarct volume was automatically calculated by multi-slice computed tomography. Disease severity was assessed using the National Institutes of Health Stroke Scale (NIHSS) at presentation, $24 \mathrm{~h}$, $72 \mathrm{~h}$ and the $28^{\text {th }}$ day. Progression was defined as an increase of more than two points in the NIHSS scores.

Results: The mean BNP levels were $284.16 \pm 382.79$ at presentation and $273.78 \pm 451.91$ at $72 \mathrm{~h}$ in the patient group, whereas the mean BNP level was $25.29 \pm 13.47$ in controls. There was a statistically significant difference between the two groups $(p<0.001)$. Differences in BNP levels among patient subgroups according to the TOAST and OCSP classifications were not statistically significant ( $p=0.534, p=0.943$, respectively). There was no significant correlation between plasma BNP level and infarct volume or NIHSS scores $(p=0.5, p=0.07)$. A positive correlation was found between BNP levels and the length of the hospitalization period ( $p=0.03$ and $r=0.33$ ). There was no statistically significant relationship between elevated plasma BNP levels and progression of disease $(p=0.08)$.

Conclusions: Plasma BNP levels were increased in the acute phase of stroke; therefore, BNP could be used as a biomarker for morbidity and mortality, even in patients without cardiac failure.

Key words: brain natriuretic peptide, stroke, prognosis, diagnosis, biomarker.

\section{Introduction}

Stroke is defined as 'the cessation of focal (sometimes global, depending on hemostatic changes) cerebral function and rapidly worsening clinical symptoms, lasting more than 24 hours (h) and without any obvious vascular cause' [1]. Stroke is an acute and rapidly progressing event. It is common worldwide, and it can cause serious morbidity and mortality. One in five strokes results in death [2].

Brain natriuretic peptide (BNP) is a neurohormone which is synthesized by cardiomyocytes in a short time and in large quantities [3]. Brain natriuretic peptide is released after the stimulation of cardiomyocytes in

\author{
Corresponding author: \\ Dilcan Kotan \\ Department of Neurology \\ Faculty of Medicine \\ Sakarya University \\ 25050 Sakarya, Turkey \\ Phone: 4882143030 \\ E-mail: dilcankotan@ \\ yahoo.com
}


response to volume or pressure overload. The cardiac wall stress stimulates the synthesis and release of BNP [3]. In addition, it is known that BNP is secreted in the brain, primarily the hypothalamus, and this release is induced by cerebral ischemia [4-6]. Brain natriuretic peptide is released in the event of excessive salt and water retention or an increase in blood pressure. Moreover, BNP protects the heart against acute volume load by reducing systemic vascular resistance and plasma volume [3]. It has an active role in cardiac autonomic regulation $[7,8]$.

Recently, many studies have demonstrated a relationship between elevated levels of natriuretic peptide and the acute phase of ischemic stroke [911]. Similar studies have found that increased plasma BNP levels are associated with cerebral ischemia and the development of neurological disease [12].

Using existing data on the relationship between plasma BNP levels and acute ischemic stroke, we designed an analytical study in order to assess the relationship between plasma BNP levels and disease prognosis as well as diagnostic features such as disease severity, infarct volume and infarct location.

\section{Material and methods}

We prospectively evaluated consecutive patients who presented within $24 \mathrm{~h}$ of the onset of acute ischemic stroke between May, 2013 and May, 2015 and then were followed up in the Sakarya University Neurology Department. The study was approved by the local ethics committee, and informed consent was obtained from each patient or a family member when necessary. All subjects who participated were volunteers.

In this study, 40 patients with a clinically and radiologically confirmed diagnosis of stroke and 30 healthy subjects were included. Healthy subjects who were selected were aged between 45 and 75 years and had no chronic diseases. Patients with heart failure (known or newly identified), myocardial infarction, chronic obstructive pulmonary disease, pulmonary hypertension, pulmonary embolism, lung cancer, tuberculosis, chronic renal failure and those under hormone replacement treatment were excluded from the present study.

All patients underwent a comprehensive physical examination focused on a neurologic examination, electrocardiography (ECG), posterior-anterior (PA) chest X-ray, blood chemistry and blood count tests. The acute ischemic stroke patients underwent, as a standard practice, multi-slice computed tomography (CT), transthoracic echocardiography (TTE) and bilateral vertebral-carotid artery Doppler ultrasonography (USG). The National Institutes of Health Stroke Scale (NIHSS) scores of these patients were determined on admission as well as $24 \mathrm{~h}, 48 \mathrm{~h}$ and 28 days after stroke.
The NIHSS was used to evaluate disease severity and to determine the prognosis. Patients were classified as follows according to the NIHSS score: 1 to 8 is mild, 9 to 15 is moderate and $\geq 16$ is severe. An increase of more than two percentage points in the NIHSS score was defined as progression.

The Oxfordshire Community Stroke Project (OCSP) classification was used to determine the anatomical subtype of stroke, based on multidetector CT findings taken within $24 \mathrm{~h}$. The etiological subtype of stroke was performed according to the Trial of Org 10172 in Acute Stroke Treatment (TOAST) classification, which is based on multislice $C T$, the bilateral vertebral-carotid Doppler USG and TTE findings [13, 14].

The subjects were divided into groups as follows according to the size of the infarction observed on the CT [13]: large infarction group (> $\left.10 \mathrm{~cm}^{3}\right)$, middle infarction group $\left(4.1-10 \mathrm{~cm}^{3}\right)$ and small infarction group $\left(\leq 4 \mathrm{~cm}^{3}\right)$.

Intravenous samples were collected in $1 \mathrm{cc}$ tubes with heparin on post-stroke days one and three. The microparticle immunoassay method was used for determination of plasma BNP levels (i1000 Architect, Abbott Laboratories, Sakarya, Turkey; normal < $100 \mathrm{pg} / \mathrm{ml}$ ), and results were given in $\mathrm{pg} / \mathrm{ml}$. Infarct volume was automatically calculated with the help of multi-slice CT.

\section{Statistical analysis}

Data were analyzed using descriptive statistical methods (mean, standard deviation (SD), frequency distribution and percentage). Continuous variables are expressed as the mean \pm the SD and categorical variables are reported in percentages. Chi-squared $\left(\chi^{2}\right)$ and Fisher's tests were used to compare categorical data. Quantitative data were tested with the Kolmogorov-Smirnov test before analysis, whether they were normally distributed or not. Comparison of two independent groups, $\chi^{2}$ and Mann-Whitney test were used. The Kruskal-Wallis test was used in order to compare more than two groups. A dependent $t$-test was used to compare the addicted group. A non-parametric Spearman test was used for correlation analysis. Results were evaluated with the $95 \%$ confidence interval, and values of $p<0.05$ were considered to be significant. The Statistical Package for Social Sciences (SPSS Inc., Chicago 2009, originally released as PASW Statistics for Windows, Version 18.0) program was used.

\section{Results}

A total of 40 patients (18 men, 26.6\%) were enrolled in the present study. The mean age was $66.03 \pm 9.95$ years. The mean \pm SD of the total 
group NIHSS scores obtained on admission and at $24 \mathrm{~h}, 48 \mathrm{~h}$ and 28 days post-stroke were 10.37 $\pm 9.09,9.05 \pm 9.06$ and $8.65 \pm 8.87$, respectively. The median plasma BNP concentration for the entire group on admission and $72 \mathrm{~h}$ after stroke was $284.16 \pm 382.79 \mathrm{pg} / \mathrm{ml}$ and $273.78 \pm 451.9$, respectively. The mean age of the healthy control group was $51.54 \pm 6.28$ years, and the mean plasma BNP level for the stroke group was $25.29 \pm 13.47 \mathrm{pg} / \mathrm{ml}$. The primary outcome measurement was death. Mortality data were obtained according to the results observed during hospitalization.

Four (10\%) patients died during hospitalization. The causes of death were brain edema and cerebral herniation.

Table I shows the baseline characteristics of the patient group and the control group. Age, prevalence of hypertension, history of diabetes mellitus (DM), admission glucose and $\mathrm{HbA}_{1 c}$ levels were significantly higher in the patient group than in the control group. However, no statistically significant differences were found in terms of gender, atrial fibrillation, history of stroke or use of alcohol.

In the ischemic stroke group, mean plasma BNP levels on admission and after $72 \mathrm{~h}$ were 284.16 \pm 382.79 and $273.78 \pm 451.9$, respectively. In the control group, the mean level of plasma BNP was $25.29 \pm 13.47$. The mean \pm SD of the plasma BNP level of the ischemic stroke group was significantly higher than that of the control group $(p<0.001)$. In the stroke group, plasma levels of
BNP on admission were higher than at $72 \mathrm{~h}$. But the differences of BNP levels on admission and $72 \mathrm{~h}$ was no statistically significant ( $p=0.1$, Table II).

The etiologic classifications according to TOAST are presented in Figure 1, and the anatomical classifications according to OCSP are presented in Figure 2. Brain natriuretic peptide levels of ischemic stroke subtype according to the TOAST and OCSP classifications did not differ significantly between the groups ( $p=0.534, p=0.943$, respectively) (Table III).

Plasma BNP level was not correlated with NIHSS score or infarct volume, but it was positively correlated with duration of hospitalization ( $p=0.5, p=0.07, p=0.03$ and $r=0.33$, respectively). Moreover, plasma BNP levels on admission was associated with death during hospitalisation. NIHSS score, glucose levels, arterial tension, presence of atrial fibrillation on admission and infarct volume did not differ significantly among subgroups.

Plasma BNP level was found to be positively correlated with mean arterial pressure (MAP) ( $p$ $=0.01, r=0.3)$. However, in ischemic stroke patients, plasma BNP levels showed no statistically significant correlation with hypertension. In this study, it was notable that the presence of atrial fibrillation did not significantly affect the level of BNP. ECO examination revealed that BNP levels were only significantly correlated with left atrial enlargement $(p=0.03)$. Left atrial enlargement

Table I. Risk classification of control and patient groups

\begin{tabular}{|c|c|c|c|}
\hline Parameter & Patient group $(n=40)$ & $\begin{array}{l}\text { Control group } \\
\quad(n=30)\end{array}$ & $P$-value \\
\hline Gender (F/M) & $22(55 \%) / 18(45 \%)$ & $22(73.3 \%) /(26.6 \%)$ & $0.101^{\star * \star}$ \\
\hline Hypertension & $9(22.5 \%)$ & $2(0.6 \%)$ & $<0.001^{* * *}$ \\
\hline Diabetes & $10(25 \%)$ & $0(0 \%)$ & $0.004^{* * *}$ \\
\hline Glucose & $129.47 \pm 58.01$ & $94.03 \pm 21.43$ & $<0.001^{* *}$ \\
\hline $\mathrm{HbA}_{1 \mathrm{c}}$ & $6.81 \pm 1.57$ & $5.66 \pm 0.42$ & $0.03^{\star *}$ \\
\hline Smoking & $12(23.1 \%)$ & $4(13.3 \%)$ & $0.20^{*}$ \\
\hline Hyperlipidemia & $19(47.5 \%)$ & $7(23.3 \%)$ & $0.27^{\star * \star}$ \\
\hline Atrial fibrillation & $6(15 \%)$ & $0(0 \%)$ & $0.03^{\star * *}$ \\
\hline Stroke history & 29 (72.5\%) & $2(0.6 \%)$ & $0.002^{* \star *}$ \\
\hline
\end{tabular}

Table II. Plasma BNP levels of patient and control groups

\begin{tabular}{|lccc|}
\hline BNP & $\begin{array}{c}\text { Patient group }(n=40) \\
\text { Mean } \pm \text { SD }\end{array}$ & $\begin{array}{c}\text { Control group }(n=30) \\
\text { Mean } \pm \text { SD }\end{array}$ & $P$-value \\
\hline Admission & $284.16 \pm 382.79$ & $25.29 \pm 13.47$ & $<0.001^{\star \star}$ \\
\hline $72 \mathrm{~h}$ & $273.78 \pm 451.91$ & $25.29 \pm 13.47$ & $<0.001^{\star \star}$ \\
\hline
\end{tabular}




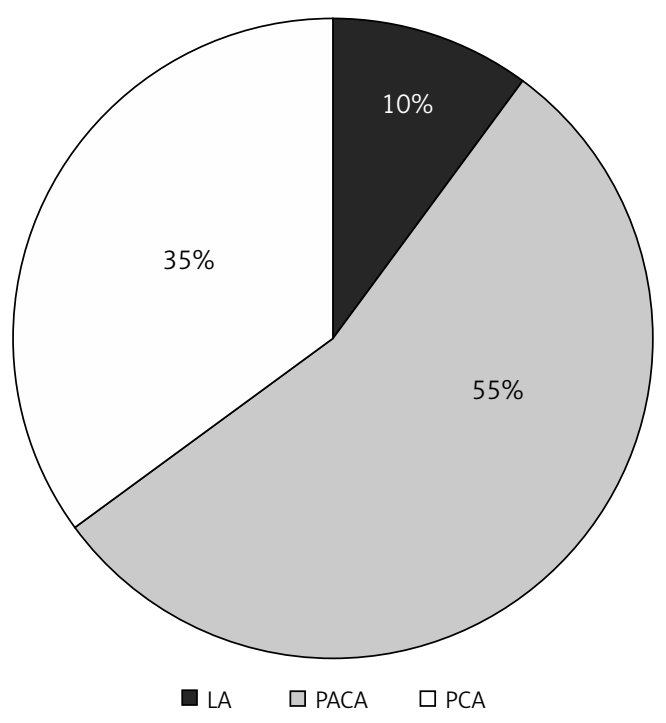

Figure 1. The distribution of patient according to OXFORD classification

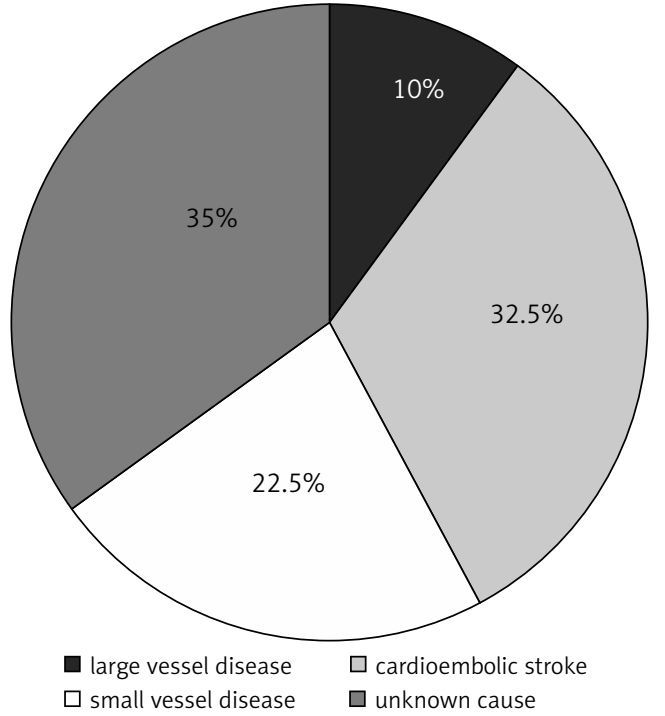

Figure 2. The distribution of patient according to TOAST classification

Table III. Relationship between BNP levels in the subset of ischemic stroke according to TOAST and OCSP classification

\begin{tabular}{|lcccc|}
\hline Variable & \multicolumn{3}{c|}{ BNP } & P-value \\
\cline { 2 - 4 } & Min. & Max. & Mean \pm SD & \\
\hline TOAST: & & & & \\
\hline Large vessel disease $(n=4)$ & 75.00 & 302.00 & $142.90 \pm 10$ & $0.534^{*}$ \\
\hline Cardioembolic stroke $(n=13)$ & 86.40 & 2097.30 & $375.20 \pm 53$ & \\
\hline Small vessel disease $(n=9)$ & 50.40 & 773.40 & $163.87 \pm 22$ & \\
\hline Unknown cause $(n=14)$ & 61.00 & 1001.90 & $317.32 \pm 33$ & \\
\hline OCSP: & & & $231.82 \pm 191.74$ & $0.943^{* *}$ \\
\hline PACA $(n=22)$ & 61.0 & 773.40 & $396.37 \pm 592.64$ & \\
\hline PCA $(n=14)$ & 81.00 & 2097.30 & $179.30 \pm 179.12$ & \\
\hline LA $(n=4)$ & 50.40 & 444.30 & 0 & \\
\hline TACA $(n=0)$ & 0 & 0 & & \\
\hline
\end{tabular}

${ }^{*} \chi^{2}$ test, ${ }^{* *} K r u s k a l-W a l l i s . P A C A-p a r t i a l$ anterior circulation infarction, $P C A-$ posterior circulation infarcts, lacunar infarction (LA) and total anterior circulation infarction (TACA).

was associated with high NIHSS scores on admission, but no significant relationship between these factors was identified after 28 days. Although plasma BNP levels were statistically significantly different from high-density lipoprotein cholesterol (HDL-C) levels, there was no significant difference between plasma BNP levels and low-density lipoprotein (LDL), total cholesterol (tcol) or triglycerides (TG).

\section{Discussion}

Stroke is an acute and rapidly progressive disease that leads to $50 \%$ of emergency neurological presentations, and $15 \%$ of chronic care services are stroke patients [15]. Stroke is the number one in disability-causing illnesses, and it is the fourth most common cause of mortality worldwide [16]. Imaging methods play an important role in the diagnosis of stroke and the determination of stroke subtypes, but they might also be significant after a certain period of time. The time restrictions associated with imaging have led researchers to consider other possible methods of rapid diagnosis, such as using biomarkers. In our study, we aimed to investigate whether plasma BNP levels were likely to be a biomarker in the diagnosis of ischemic stroke, especially cardioembolic subtype, and if so, whether this could be used to predict disease severity and prognosis. 
Diagnosis of acute ischemic stroke is determined through evaluating the patient's history, neurological examination and imaging techniques. Studies have shown that better image quality can be obtained with multi-slice spiral CT and that this method results in fewer bone artifacts and requires less time than other $\mathrm{CT}$ techniques. It also provides more accurate information for volume calculations by providing three-dimensional images [17-19]. In our study, we increased the reliability of localization of the lesion and the infarct volume calculation by using this new imaging method.

After the diagnosis of stroke, determination of stroke subtypes is the next crucial step for predicting prognosis, choosing treatment strategies, deciding on the appropriate discharge phase and developing a strategy of relapse prevention. Currently, stroke subtype is determined using the aid of brain $C T$, magnetic resonance (MR) imaging, $M R$ angiography, ECG and TTE. In our study, we carried out stroke typing with the help of brain CT, ECG and TTE.

The etiologic subtypes of ischemic stroke are as follows: large vessel disease, cardioembolic stroke and small vessel disease or stroke of unknown cause according to the TOAST classification [14].

The anatomic subtypes of ischemic stroke are as follows: partial anterior circulation infarction (PACA), posterior circulation infarcts (PCA), lacunar infarction (LA) and total anterior circulation infarction (TACA) according to the OCSP classification [13]. In our study, we used the TOAST and OCSP classifications.

Imaging methods play a considerable role in the diagnosis of ischemic stroke and the determination of stroke subtype; however, it takes time to gain access to and use these methods. Time limitations have led researchers to consider other possible methods for rapid diagnosis.

Recently, several studies have suggested that there is a relationship between the acute phase of ischemic stroke and the elevation of natriuretic peptides $[9,10]$. In our study, plasma BNP levels during the first 24 and $72 \mathrm{~h}$ were significantly higher in the stroke group than in the control group $(p<0.001)$. Thus, our findings are consistent with the existing studies.

Plasma BNP level is a parameter used in the evaluation of cardiac failure [20]. In our study, many factors that lead to increasing plasma BNP levels were adopted as exclusion criteria. As a result, patients suffering from cardiac failure were excluded; this is a difference between our study and previous studies. In our study, patients with no history but with cardiac failure determined after echocardiography were excluded. These patients had higher BNP levels than the other stroke patients. The sample group was too small and statistical analysis could not be done [21].

It is thought that BNP has an important role in stroke, especially in hemodynamic regulation in the acute phase of ischemic stroke [22-24]. Our study revealed a positive correlation between plasma BNP and MAP ( $p=0.016, r=0.3)$. There was no statistically significant association between plasma BNP levels and presence of hypertension in the stroke group. Thus, our study confirms the relationship between BNP levels and acute hemodynamic changes. In addition, our study's results appear to indicate that the relationship between BNP levels and hemodynamic changes may persist for up to $72 \mathrm{~h}$.

Shibazaki et al. suggested that plasma BNP levels are significantly higher in patients with cardioembolic stroke than in those with large vessel, small vessel and other stroke [25]. Moreover, Kim et al. stated that plasma BNP levels are elevated in cardioembolic stroke, large vessel atherosclerosis, and small vessel stroke (types of stroke are listed in order of decreasing associated plasma BNP levels) [26]. In our study, there was no significant difference between plasma BNP levels and stroke subgroups according to the TOAST classification. Heart failure is a major cause of cardioembolic stroke. We consider that the exclusion of patients with cardiac insufficiency might have had an effect on the statistical results.

We did not collect enough data to verify the relationship between plasma BNP levels and in farct volume reported in the literature $[11,27]$ However, Kim et al. suggested that there was a significant positive correlation between plas ma BNP levels and infarct volume in the first $72 \mathrm{~h}$ [28]. Similarly, Chen et al. stated that there was a significant positive correlation between a large infarct volume $\left(>10 \mathrm{~cm}^{3}\right)$ and plasma BNP levels in the first $72 \mathrm{~h}$ [29]. In our study, there was no significant correlation between plasma BNP levels and infarct volume in the stroke group. Studies showing a positive correlation included patients with cardiac failure; however, these patients were excluded from our study, and this might have contributed to the different results. Our study results suggest that there was no significant correlation between BNP levels and infarct volume.

When patient and control groups were compared, there were statistically significant differences found in terms of risk factors, glucose level, diabetes, $\mathrm{HbA}_{1 \mathrm{c}}$ and presence of hypertension. No statistically significant differences were found for gender, atrial fibrillation, stroke or alcohol consumption (Table I). Several studies have suggested that plasma BNP levels are associated with the development of cerebral ischemia and neurological deficits [12] Moreover, some studies have shown that plasma BNP level is predictive of NIHSS score on admission 
$[11,30]$. For example, Chen et al. observed a significant correlation between NIHSS score on admission and plasma BNP levels [29]. Shibazaki et al. reported a significant relationship between the plasma BNP level in the first $24 \mathrm{~h}$ and NIHSS score on admission [25]. Our study results did not demonstrate a significant correlation between plasma BNP levels and NIHSS score on admission.

Brain natriuretic peptide levels in the stable phase of acute ischemic stroke can be used for the prediction of short-term hospital discharge [31]. Brain natriuretic peptide levels are also a strong predictor of long-term mortality [10]. Therefore, it is understood that increased BNP levels in stroke patients not only show cardioembolic etiology but they can also be an indicator of long-term poor prognosis, including hospital mortality [30, 32, 33]. However, our study suggests that there was no statistically significant relationship between increased plasma BNP levels and the progression of disease $(p=0.08)$.

Recently, studies have reported that increased plasma BNP levels are associated with mortality in the acute phase of stroke [10, 34]. Mäkikallio et al. suggested that plasma BNP levels were independently associated with mortality after hospital admissions for stroke [34]. In addition, Shibazaki et al. reported that plasma BNP level is an important predictor of hospital mortality in patients with acute stroke [33]. Our study results suggest that plasma BNP level has an independent predictive value in stroke.

In conclusion, a biomarker that can be applied in stroke diagnosis and typing will provide advantages in terms of both ease and speed of diagnosis. Researchers all over the world are trying to discover such a biomarker. We attempted to use BNP as a parameter for this purpose, and we achieved significant results by determining a correlation between BNP levels and mortality and prognosis. We believe that this biomarker can be easily, cheaply and usefully applied in clinical practice.

\section{Conflict of interest}

The authors declare no conflict of interest.

\section{References}

1. Cerebrovascular Disorders: A Clinical and Research Classification. No: 43. WHO offset publication Geneva, Switzerland 1978.

2. Townsend N, Wickramasinghe K, Bhatnagar P, et al. Coronary heart disease statistics. A compendium of health statistics 2012 edition. British Heart Foundation, October 2012; 72.

3. Azzazy HM, Cristenson RH. B-type natriuretic peptide: physiologic role and assay characteristics. Heart Fail Rev 2003; 8: 315-20.
4. Takahashi K, Totsune K, Sone M, et al. Human brain natriuretic peptide-like immunoreactivity in human brain. Peptides 1992; 13: 121-3.

5. Nogami M, Shiga J, Takatsu A, Endo N, Ishiyama I. Immunohistochemistry of atrial natriuretic peptide in brain infarction. Histochem J 2001; 33: 87-90.

6. Giuffrida R, Bellomo M, Polizzi G, Malatino LS. Ischemia-induced changes in the immunoreactivity for endothelin and other vasoactive peptides in the brain of the Mongolian gerbil. J Cardiovasc Pharmacol 1992; 20 (Suppl 12): S41-4.

7. Herring N, Zaman JA, Paterson DJ. Natriuretic peptides like NO facilitate cardiac vagal neurotransmission and bradycardia via a cGMP pathway. Am J Physiol Heart Circ Physiol 2001; 281: H2318-27.

8. Thomas CJ, Woods RL. Guanylyl cyclase receptors mediate cardiopulmonary vagal reflex actions of ANP. Hypertension 2003; 41: 279-85.

9. Shibazaki K, Kimura K, Okada Y, et al. Plasma brain natriuretic peptide as an independent predictor of in-hospital mortality after acute ischemic stroke. Intern Med 2009; 48: 1601-6.

10. Jensen JK, Atar D, Kristensen SR, Mickley H, Januzzi JL Jr. Usefulness of natriuretic peptide testing for long-term risk assessment following acute ischemic stroke. Am J Cardiol 2009; 104: 287-91.

11. Di Angelantonio E, De Castro S, Toni D, et al. Determinants of plasma levels of brain natriuretic peptide after acute ischemic stroke or TIA. J Neurol Sci 2007; 260: 139-42.

12. Sviri GE, Shik V, Raz B, Soustiel JF. Role of brain natriuretic peptide in cerebral vasospasm. Acta Neurochir 2003; 145: 851-60.

13. Bamford J, Sandercock P, Dennis M, Burn J, Warlow C. Classification and natural history of clinically identifiable subtypes of cerebral infarction. Lancet 1991; 337: 1521-6.

14. Adams HP Jr, Bendixen BH, Kappelle LJ, et al. Classification of subtype of acute ischemic stroke. Definitions for use in a multicenter clinical trial. TOAST. Trial of Org 10172 in acute stroke treatment. Stroke 1993; 24: 35-41.

15. Waxman SG. Correlative Neuroanatomy. 24 ed. Lange Medical Books, New Haven 1999; 68-172.

16. Schwamm LH, Audebert HJ, Amarenco P, et al.; American Heart Association Stroke Council; Council on Epidemiology and Prevention; Interdisciplinary Council on Peripheral Vascular Disease; Council on Cardiovascular Radiology and Intervention. Recommendations for the implementation of telemedicine within stroke systems of care: a policy statement from the American Heart Association. Stroke 2009; 40: 2635-60.

17. Alberico RA, Loud P, Pollina J, Greco W, Patel M, Klufas $R$. Thick-section reformatting of thinly collimated helical CT for reduction of skull base-related artifacts. AJR Am J Roentgenol 2000; 175: 1361-6.

18. Dorenbeck U, Finkenzeller T, Hill K, Feuerbach S, Link J. [Volume-artifact reduction technique by spiral $\mathrm{CT}$ in the anterior, middle and posterior cranial fossae. Comparison with conventional cranial CT]. Rofo 2000; 172: 342-45.

19. Jones TR, Kaplan RT, Lane B, Atlas SW, Rubin GD. Single- versus multi-detector row CT of the brain: quality assessment. Radiology 2001; 219: 750-5.

20. Tsutamoto T, Wada A, Maeda K, et al. Attenuation of compensation of endogenous cardiac natriuretic peptide system in chronic heart failure: prognostic role of plasma brain natriuretic peptide concentration in patients with chronic symptomatic left ventricular dysfunction. Circulation 1997; 96: 509-16. 
21. Bibbins-Domingo K, Gupta R, Na B, Wu AH, Schiller NB, Whooley MA. N-terminal fragment of the prohormone brain-type natriuretic peptide (NT-proBNP), cardiovascular events, and mortality in patients with stable coronary heart disease. JAMA 2007; 297: 169-76.

22. Levin ER, Gardner DG, Samson WK. Natriuretic peptides. N Engl J Med 1998; 339: 321-8.

23. Bonow RO. New insights into the cardiac natriuretic peptides. Circulation 1996; 93: 1946-50.

24. Rossi A, Enriquez-Sarano M, Burnett JC Jr, Lerman A, Abel $M D$, Seward JB. Natriuretic peptide levels in atrial fibrillation. A prospective hormonal and Doppler-echocardiographic study. J Am Coll Cardiol 2000; 35: 1256-62.

25. Shibazaki K, Kimura K, Iguchi Y, Okada Y, Inoue T. Plasma brain natriuretic peptide can be a biological marker to distinguish cardioembolic stroke from other stroke types in acute ischemic stroke. Intern Med 2009; 48: 259-64.

26. Kim SH, Lee JY, Park SH, et al. Plasma B-type natriuretic peptide level in patients with acute cerebral infarction according to infarction subtype and infarction volume. Int J Med Sci 2013; 10: 103-9.

27. Nakagawa K, Yamaguchi T, Seida M, et al. Plasma concentrations of brain natriuretic peptide in patients with acute ischemic stroke. Cerebrovasc Dis 2005; 19: 157-64.

28. Richards AM, Lainchbury JG, Nicholis MG, Cameron AV, Yandle TG. Dendroaspis natriuretic peptide: endogenous or dubious. Lancet 2002; 359: 5-6.

29. Chen X, Zhan X, Chen M, et al. The prognostic value of combined NT-pro-BNP levels and NIHSS scores in patients with acute ischemic stroke. Intern Med 2012; 51: 2887-92.

30. Montaner J, Perea-Gainza M, Delgado P, et al. Etiologic diagnosis of ischemic stroke subtypes with plasma biomarkers. Stroke 2008; 39: 2280-7.

31. Sharma JC, Ananda K, Ross I, Hill R, Vassallo M. N-terminal proBrain natriuretic peptide levels predict shortterm poststroke survival. J Stroke Cerebrovasc Dis 2006; 15: 121-7.

32. Grau AJ, Weimar C, Buggle F, et al. Risk factors, outcome, and treatment in subtypes of ischemic stroke: the German stroke data bank. Stroke 2001; 32: 2559-66.

33. Shibazaki K, Kimura K, Iguchi Y, Aoki J, Sakai K, Kobayashi K. Plasma brain natriuretic peptide predicts death during hospitalization in acute ischaemic stroke and transient ischaemic attack patients with atrial fibrillation. Eur J Neurol 2011; 18: 165-9.

34. Mäkikallio AM, Mäkikallio TH, Korpelainen JT, et al. Natriuretic peptides and mortality after stroke. Stroke 2005; 36: 1016-20. 